\title{
Pasi Valtonen* Semantic innocence and Kaplanian inferences
}

https://doi.org/10.1515/sats-2019-0006

Abstract: The core of Christopher Hom and Robert May's semantic innocence is the thesis that ethnic slurs have empty extensions. Thereby, a slurring term makes any non-negated slurring sentence false. At the same time, Hom and May emphasise that the most important task in the study of slurs is to explain non-xenophobic understanding of slurs. In this paper, I argue that there is a conflict between the two claims. I show this with Kaplanian inferences, which, in my view, are crucial for the non-xenophobic understanding. After all, slurs offend us because we understand them.

Keywords: ethnic slurs, derogation, Kaplanian inferences, validity, truth preservation

\section{Introduction}

In their "Moral and Semantic Innocence", Hom and May (2013: 293-313) argue that a fundamental question for any account of ethnic and racial slurs is

How can a competent, rational speaker know the meaning of a slur without being committed to, or even complicit with, xenophobic attitudes?

I very much agree that this is an important question with slurs. After all, slurs offend us because we understand them. As an answer to their question, Hom and May offer semantic innocence. The outline of semantic innocence is captured with the following claims:

(i) No Germans are Boches. ${ }^{1}$

(ii) There are no Boches.

(iii) There are Germans.

$\overline{1}$ In this paper, I use somewhat outdated slurs that are less offensive but still serve the illustrative purpose.

*Corresponding author: Pasi Valtonen, Philosophy, Tampere University, Tampere, Finland, E-mail: pablo.valtonen@gmail.com

https://orcid.org/0000-0002-9470-1486 
The basis of semantic innocence is the idea that although the term 'German' denotes the set of German people, the corresponding slurring term 'Boche' does not denote anything. Hence, everything expressed with this term comes out false. Needless to say, this concerns only unnegated sentences as (ii) is part of the package and, as such, a profound truth, according to semantic innocence.

Semantic innocence relies on moral innocence, which is the thesis that "there are no morally evaluable traits (good or bad) that are heritable on the basis of race, gender, sexual orientation, and the like”. Hom and May continue: “There are no kikes because there is no one who ought to be the object of negative moral evaluation just because they are Jewish" (Hom and May 2013: 295). Semantic innocence, the thesis that slurring utterances are false, follows from moral innocence, from the thesis that no one should be treated negatively on the basis of their ethnicity and so on.

In this paper, I show that there is a conflict between Hom and May's aims and their means of achieving this goal. That is, a conflict between their main aim to explain the non-xenophobic understanding of slurs and the main tenet of semantic innocence that all unnegated slurring sentences are false. In general, a view of slurs has to explain the understanding of slurs and it has to explain derogation involved with slurs. These demands are connected. We find slurs offensive because we understand them. I show that Hom and May explain derogation at the expense of understanding. Specifically, even though Hom and May's question concerning non-xenophobic understanding is critical, I do not think that semantic innocence is the right answer. In the course of the argumentation, I introduce Kaplanian inferences, which, in my opinion, are crucial concerning the non-xenophobic understanding. I go on to elaborate Kaplanian inferences with Stefano Predelli's development of Kaplan's initial thoughts about inferences containing expressive elements. Predelli has specifically discussed slurs in this context. I take it that his refinement of Kaplan's thoughts highlights truth preservation in Kaplanian inferences and that semantic innocence cannot accommodate truth preservation.

\section{Semantic innocence}

Hom and May introduced PEJ (short for pejorative), a semantic tool to track the behaviour of slurs. Intensionally, it is something like

PEJ: (Individual) $\mathrm{x}$ ought to be the target of negative moral evaluation because of being (a member of) $\mathrm{X}$. 
When PEJ is applied to 'German', we get insight into the meaning of 'Boche', which is something like:

Boche: $\mathrm{x}$ ought to be the target of negative moral evaluation because of being German. ${ }^{2}$

If we have a neutral term, such as 'German', then Hom and May's lexical analysis of 'Boche' is PEJ(German). PEJ denotes a second-level function that applies to a first-level concept, i.e. to predicates like ethnicity, religion and so on. Generally, in intensional semantics, the intension of a term is taken to be the meaning of the term, which then determines the extension of the term. For example, the intension or the meaning of 'Bachelor' could be paraphrased as an unmarried man. This then yields the set of unmarried men as the extension of 'Bachelor'. Similarly, the intension or the meaning of PEJ is ' $x$ ought to be the target of negative moral evaluation because of being X', but the crucial observation is that no one deserves to the target of negative moral evaluation. Hence, the intension of PEJ determines the extension to be a function, which takes the extension of the original predicate to an empty set. As Hom and May point out, the fact that "complex pejorative concepts are constant functions mapping individuals to falsity is the realization of the null extension thesis" (Hom and May 2013: 298).

The type of pejorative concepts is comparable to the type of predicate modifiers, $\langle\langle e \rightarrow t\rangle \rightarrow\langle e \rightarrow t\rangle\rangle$. Ordinarily, a predicate modifier takes the extension of a predicate expression to a subset, as in " $x$ is a strong horse". The set of strong horses is a subset of horses, but sometimes, the modifier can take the extension to a completely different set, as in " $x$ is a toy horse". The extension of a toy horse is not a subset of horses but, presumably, a subset of toys. In this spirit, Hom and May suggest that PEJ should be compared with expressions like 'fictional' or 'magical'. These take the extension (whatever it is) to an empty set. Surely, there are horses but there are no magical horses. The extension of the expression 'magical(horse)' is empty. Analogously, the extension of PEJ(German) is empty

2 This view is somewhat different from Hom's earlier view. In his "The Semantics of Racial Epithets", Hom says that the meaning of slurs includes prescriptions to discrimination on the basis of (inaccurate) descriptions (Hom 2008: 431).The meaning of 'Frog' could then be characterised as

$\mathrm{x}$ ought to be placed in one of the lesser tables in a restaurant because of having vulgar eating habits because of being French.

In their joint paper, Hom and May have simplified the meaning of slurs. Despite the differences in the details, the general claim of semantic innocence is still the same. Hom defends the thesis that non-negated slurring sentences are systematically false. 
and that is why unnegated atomic sentences containing PEJ come out false. ${ }^{3}$ (Hom and May 2013: 293-313; see also Hom 2008: 416-440.)

From this point of view, Hom and May go on to explain the non-xenophobic understanding of slurs. Importantly, the consequence of semantic innocence is an asymmetry between the xenophobic and the non-xenophobic understanding. Hom and May are quite explicit about this:

Racists believe that pejorative terms have non-null extensions; they believe that there are kikes, chinks, and the like. [...] But they are wrong, and they are wrong about what the word 'kike' means. They may grasp the same underlying concept as a non-racist, yet nevertheless they are linguistically incompetent as that knowledge does not issue the right truth-conditions for sentences containing pejoratives. In contrast, non-racists do properly know the meanings of pejorative terms, and assign the proper truth-conditions. (Hom and May 2013: 297)

Racists and xenophobes are obviously wrong about the world, but it is less obvious that they are also incompetent speakers. On the contrary, it seems that the racist and the xenophobes are rather capable of expressing their racist and xenophobic attitudes. In the following, I will argue that this asymmetry between xenophobic and non-xenophobic linguistic understanding is very problematic. First and foremost, it is problematic for the non-xenophobic understanding. Kaplanian inferences highlight this.

\section{Kaplanian inferences}

On the basis of David Kaplan's “The Meaning of 'Ouch' and 'Oops”, two instances of Kaplanian inferences involving slurs can be formed:

(1) Max is a Boche. Thus, Max is a German.

(2) Max is a German. Thus, Max is a Boche. ${ }^{4}$

3 Both qualifications, unnegated and atomic, are important. Hom and May acknowledge that negated slurring sentences, like "Max is not Frog, He is French" are true. Also, the falsity has to be contained only to atomic sentences. Slurring sentences can appear in true complex sentences such as "Max is a Frog and Fritz is a Boche or snow is white", which is true when disjunction is the principal connective. According to semantic innocence, the universally quantified sentence "All Frogs are French" $(\forall x(F r o g(x) \rightarrow$ French $(x)))$ is also true since the false antecedent is enough to secure the truth of the whole implication.

4 Goes without saying that (1) and (2) correspond with Kaplan's famous examples

$\left(1^{\star}\right)$ That damn Kaplan is promoted. Thus, Kaplan is promoted.

$\left(2^{\star}\right)$ Kaplan is promoted. Thus, that damn Kaplan is promoted. (Kaplan 2004, 4) 
In my view, (1) reflects non-xenophobic understanding and (2) reflects xenophobic understanding. Because Hom and May concentrate on explaining the non-xenophobic understanding, the focus here is also on (1). Imagine that nonxenophobic Mary asks xenophobic Jill about a recent party:

(3) a. Mary: Jill, do you know who were at the party yesterday?

b. Jill: Yes, there were a few Boches and a Frog.

Later, Mary is asked about the party and she replies:

(4) Yes, there were a few Germans and a Frenchman.

This is analogous to (1). Even though the point is very simple, (4) illustrates the non-xenophobic understanding of slurs, as it shows that a non-xenophobic speaker can extract semantic information from slurring utterances. The question is how a non-xenophobic speaker can do this. A natural explanation is that the slurring term and its neutral counterparts are coextensional. This is also Kaplan's point. He seems to think that (1) and (2) are truth-preserving. ${ }^{5}$ Yet Kaplan claims that (1) is a valid inference and (2) is an invalid inference. On the basis of this intuition, Kaplan makes a rather radical suggestion that the validity of semantic entailment is not based on truth preservation but on what he calls information delimitation. Kaplan characterises information delimitation in the following way: For a semantic argument to be valid, there must be no information in the conclusion that is not already contained in the premises. According to Kaplan, (2) violates this constraint. Even though the truth-conditional or -propositional content is the same in the premise and in the conclusion in (2), there is an "intrusion of information" made with the expressive element of 'Frog'. The intrusion displays the speaker's xenophobic attitude (Kaplan 2004: 5-6).

Admittedly, at this stage, the ideas presented in this section are vague, but the next sections are devoted to making the ideas more specific. Namely, three questions are addressed: First, what exactly is involved with truth preservation in (1) and (2)? Second, what does semantic innocence have to do with truth preservation and information delimitation? Finally, what exactly is involved with and how plausible is the notion of validity based on information delimitation?

5 Again, he does not say specifically that slurring inferences like (1) and (2) are truth-preserving. Rather, he says that $\left(1^{\star}\right)$ and $\left(2^{\star}\right)$ are truth-preserving but the received view of Kaplan's thoughts is that the truth-conditional indifference also applies to slurs and their neutral counterparts (see Section 5 for elaboration). 
The upshot of the discussion is that semantic innocence struggles to explain the non-xenophobic understanding.

\section{Semantic innocence and Kaplanian inferences}

\subsection{Validity as truth preservation}

It seems that there is a missing premise in (1) and (2), and to explicate truth preservation, the missing premise has to be added. The missing premise involves the idea of truth-conditional indifference. In other words, there has to be a statement of the coextensionality between the 'German' and the 'Boches' in the premises:

(5) Max is a Boche $\&$ Boche $=$ German. Thus, Max is a German.

(6) Max is a German \& Boche $=$ German. Thus, Max is a Boche.

Here we can see that the inferences really are truth-preserving. In (5) and (6) the set of interpretations of the premises is a subset of the interpretations of the conclusion. It is equally obvious that semantic innocence does not pass this test. The central claim of semantic innocence is that slurring terms and their neutral counterparts differ extensionally. Hence, we have the following inferences:

(7) Max is a Boche $\&$ Boche $\neq$ German. Thus, Max is a German.

(8) Max is a German \& Boche $\neq$ German. Thus, Max is a Boche.

Even though these inferences look odd and they surely are invalid, the point of (7) and (8) is to show what Kaplanian inferences look like when claim (ii) of semantic innocence is added to the mix; claim (ii) being that no 'Boches' are Germans. Here our concentration is on (7) as that is the one that reflects non-xenophobic understanding. Given that semantic innocence subscribes to the asymmetry between non-xenophobic and xenophobic understanding, it might be that (8) just reflects the xenophobic incompetency. The problem for semantic innocence is then (7). Since the premises now include claim (ii) that no Germans are Boches, we cannot infer from "Max is a Boche" that "Max is German". Perhaps, this was a bit obvious at the outset, but the more interesting question is whether semantic innocence can explain Mary's understanding in (4) with the notion of validity based on information delimitation. 


\subsection{Validity as information delimitation}

It seems that if semantic innocence has any fighting chance, validity has to be based on information delimitation that has very little to do with truth preservation. If indeed validity has little to with truth and all to do with limiting the flow of information, then one might think that (1) is valid even according to semantic innocence as the propositional content

(9) Max is a PEJ(German).

is more complex than the content of

(10) Max is a German.

Kaplan's characterisation of information delimitation is that there must not be any information in the conclusion that is not already in the premises. Presumably, inference from (9) to (10) could satisfy Kaplan's characterisation as (10) conveys less information than (9). This train of thought might even cohere with Hom and May's proposal that the derogatory part of a slurring concept should be compared with its characteristic mark. Using Frege's distinction between concepts and their characteristic marks, Hom and May argue that the pejorative component is a characteristic mark of a slurring concept (Hom and May 2013: 302; Frege 1964: 11-12).

However, under the current notion of validity, even

(11) $\neg$ A. Thus, A

is valid. I guess by eliminating the negation sign, you are saying less than with the negation sign, but it is also very obvious that any notion of validity that rules the above as good inference is just baffling. To me, the problem is that Kaplan's idea about information delimitation is too vague. In the next section, the notion is clarified, and while it is clarified, it becomes clear that truth preservation is crucial in inferences involving slurs.

\section{Predelli and a plea for truth}

In all fairness, Kaplan is the first one to admit that his notion of information delimitation is vague. and he goes on to give one possible interpretation for the notion. He suggests that you could construct a notion of truth that is based on information delimitation. On that notion, a sentence is true only if it is 
descriptively and expressively correct. Basically, this means that an inference has to be truth-preserving (descriptively correct) as well as expressively correct. He calls this truth-plus, and on the basis of this, we can say that a sentence is truthplus-preserving only if it satisfies both descriptive and expressive aspects in an inference, but Kaplan insists that, even in that case, validity is based on information delimitation via truth-plus-preservation. Stefano Predelli calls validity based on truth-plus-preservation validity-plus. In the following, we will see Predelli's development of Kaplan's thoughts. The upshot is that, according to Predelli, validity-plus does not work. Kaplan's idea is that there is a single notion of validity, validity-plus, but Predelli shows that there are actually two equally important notions of validity, ordinary validity based on truth preservation and expressive validity. In our present context, this result emphasises the role of ordinary truth.

Even though the differences between the slurring terms and the neutral terms are truth-conditionally irrelevant, Kaplan still thinks that the meaning of slurs differs from the meaning of their neutral counterparts. First, Kaplan introduces the idea that expressives can be paraphrased in a truth-conditional way. 'Ouch' can be paraphrased as "I am in pain now" and 'oops' as "I just witnessed a minor mishap". This idea leads to another important point. Even though expressives are not truth-conditional, the paraphrasing allows to explicate the correct use of expressives within Kaplanian semantics. The content of 'ouch' is that the speaker is in pain. The semantic information of the utterance is represented as a set of contexts in which the expressive is used correctly. The meaning of 'ouch' constrains the correct use to those contexts in which the speaker actually is in pain, not just using it to get attention. These two points are at the centre stage in Predelli's treatment of slurs.

In the Kaplanian semantics, the truth-conditional aspect of meaning depends on a notion of character. It is a function from context to a semantic value. For instance, 'Kaplan' can be represented as a constant function that yields Kaplan as the semantic value of the name in every context. In this sense, the terms 'Frog' and 'French' are character indistinguishable. Given any context of utterance, the character function yields the same semantic value, namely the set of French people (Predelli 2010: 165; Kaplan 1989: 505-507). In a nutshell, this is a sophisticated formulation of the coextensionality thesis. As a result, the truthconditional contribution of 'Frog' and 'French' is the same. Hence, the difference between 'French' and 'Frog' cannot be explained truth-conditionally. The idea that derogation is not truth-conditionally relevant is not new. Many pragmatic treatments of slurs such as conventional implicature strategy and presuppositional account also rest on the contention that derogation is not part of the truthconditional meaning (see McCready 2010: 1-57; Williamson 2009: 137-158, for conventional implicature strategy, and Cepollaro and Stojanovic 2016: 458-488, 
for presuppositional account). However, the current Kaplanian approach highlights the importance of Kaplanian inferences, as the central claim of the view is that despite the truth-conditional irrelevance, there can still be inferential differences between the slurring and the neutral statements. This combination is the distinguishing feature of the Kaplanian approach to slurs.

To make the inferential differences explicit, Predelli puts the two above points to use in his treatment of slurs. Let us discuss the idea of paraphrase first. Let the paraphrase for 'Frog' be something like

(12) I regard the French as vulgar.

Here 'I' refers to the agent of the context, i.e. to the speaker who utters the word 'Frog'. Predelli's term for the paraphrase is semantic witness, but I continue to use my chosen terminology as there is no need to complicate things (Predelli 2013: 122-123). Furthermore, the current terminology highlights the distinction with descriptive ineffability, which is the thesis that expressives cannot be paraphrased without resorting to other expressives. For example, Christopher Potts and Eric McCready adhere to ineffability of expressives (Potts 2007: 166-179; McCready 2010: 5-11.) Obviously, this makes it very difficult to paraphrase expressives in a truth-conditional way. Robin Jeshion claims that slurs especially are ineffable, as the use of slurs can be offensive even if the speaker does not know the stereotypical conceptions of the target (Jeshion 2013: 322). In contrast, Predelli's treatment does entail that the meaning of slurs convey stereotypical conceptions of the targets. I take the formation of the paraphrase to be fairly unproblematic mainly because Hom also takes it as an empirical problem, not philosophical (Hom 2008: 430-431). In any case, the paraphrase has a crucial role in explaining of the meaning of slurs. First, a context $(c)$ can be taken as an n-tuple of an agent $\left(c_{a}\right)$, a time $\left(c_{t}\right)$, a possible world $\left(c_{w}\right)$. A set of contexts of correct use (CU) for 'Frog' is then:

$$
\mathrm{CU}(\text { Frog })=\left\{c \mid \text { at } c_{t} \text { and } c_{w}, c_{a} \text { regards } \llbracket \text { Frog/French } \rrbracket \text { as vulgar }\right\}
$$

On the basis of this, a constraint on correct use can be formed:

$$
c \in \mathrm{CU}\left(\text { Frog) only if at } c_{t} \text { and } c_{w}, c_{a} \text { regards } \llbracket \text { Frog/French } \rrbracket\right. \text { as vulgar }
$$

In plain English, the constraint says that the use of 'Frog' is correct in context $c$ only if it is true in $c$ that the agent regards the French as vulgar. In general, the use of a slur is correct in a given context only if the corresponding paraphrase is true in that context (Predelli 2013: 96-99). This captures Kaplan's insight that the meaning of an expressive can be represented as a set of contexts in which the paraphrase is true. Related to the truth of the paraphrase, it is important to notice that (12) displays subjective correctness conditions for 'Frog'. It reflects 
the speaker's attitude towards the French, not how things actually are in the world (Predelli 2010: 168-169). Kaplan makes a distinction between subjective and objective correctness conditions for expressives. For example, he thinks that the correctness conditions for 'ouch' are subjective, as the correctness depends on the speaker's inner state. The correctness conditions for 'oops' are objective. The use is correct only if the speaker witnessed a minor mishap close-by (Kaplan 2004: 9-14). Similarly, Predelli makes a distinction between subjective and objective correctness conditions for slurs. He considers objective conditions for the use of slurs, such as "the French are vulgar". He nevertheless ends up rejecting this idea. The problem with objective correctness conditions is that it is hard to think of context in which this paraphrase is true. Hence, the use of slurs would always be incorrect, as the paraphrase is not true in any context. This goes against my previous point that xenophobes and racists are perfectly capable of expressing their hateful and misguided thoughts. The subjective paraphrase explains the slurring instances of Kaplanian inferences:

(13) a. Max is a Frog. Thus, Max is a Frenchman.

b. Max is a Frenchman. Thus, Max is a Frog.

(13a) is valid, while the meaning of 'Frog' precludes the validity of (13b). With the paraphrase, this can be explicated. The scheme

S \& P. Thus, $\mathrm{S}_{e x}$.

(where $\mathrm{S}$ is a non-expressive sentence and $\mathrm{S}_{e x}$ truth-conditional equivalent with an expressive element) allows to introduce the expressive element of 'Frog':

(14) Max is a Frenchman \& I regard the French as vulgar. Thus, Max is a Frog.

According to Predelli, the inference is truth-preserving as 'Frenchman' and 'Frog' are character indistinguishable. That is the only relevant point here (Predelli 2010: 169-174, 2013: 96-99, 122-123). Interestingly, the converse is not truthpreserving. Observe that

(15) Max is a Frog. Thus, Max is a Frenchman \& I regard the French as vulgar.

is not truth-preserving. To see why it is not truth-preserving, consider a context in which Max indeed is a Frenchman, but the speaker uses the slur incorrectly. That is, the speaker uses the slur without the derogatory attitude. This is a counterexample to (15), as the premise is true but the conclusion is false. The premise is true since Max is a Frenchman. However, "I regard the French as vulgar" in the conclusion is false. The 'I' refers to the speaker who uses the word 
'Frog' but because the slur is used incorrectly, the speaker does not regard the French as vulgar. Thereby, the whole conclusion is false since it is a conjunction. However, the inference is expressively valid. This observation has profound ramifications concerning Kaplan's validity-plus. To see why it is expressively valid, the first observation is that Predelli aims to formulate expressive validity in the Kaplanian spirit as validity-plus ${ }^{6}$-a notion of validity that takes into account the truth-preservation and expressive aspects of Kaplanian inferences. In this case, it is reasonable to think that any expressively valid sentence has to be also truth-preserving. For that reason, Predelli bases expressive validity on the notion of true correct use context:

A context $c$ is a true correct context for $\mathrm{S}, c \in \mathrm{TCU}(\mathrm{S})$ iff $_{\text {def }}$ true $_{c}(\mathrm{~S})$ and $c \in \mathrm{CU}(\mathrm{S})$

That is, a sentence has to be true in $c$ and its use has to be correct in $c$. From this, Predelli forms expressive validity:

An argument $\mathrm{S} \therefore \mathrm{s}$ is expressively valid iff $_{d e f} \mathrm{TCU}(\mathrm{S}) \subseteq \mathrm{TCU}(\mathrm{s})$.

It seems to me that this definition of validity is the best candidate for validity-plus. The basic idea with validity-plus, presumably, is that every plus valid argument is also truth-preserving but not the other way round. However, (15) is a counterexample to this thought. Observe that TCU(S) requires that $\mathrm{S}$ is true and that it is used correctly. This rules out contexts in which it is used incorrectly. Hence, TCU(Max is a Frog) $\subseteq$ TCU(Max is a Frenchman \& I regard the French as vulgar). Still, it is not truth-preserving in the ordinary sense. The upshot is that even the best candidate for validity-plus, i.e. Predelli's expressive validity, is not validityplus. The idea that all validity-plus arguments are also truth-preserving but not the other way round does explain slurring instances of Kaplanian inferences like (1) and (2). Both are truth-preserving in the traditional way, but Kaplan's idea is that only (1) satisfies validity-plus. Indeed, in (1) and (2), this is the case but once you dig a bit deeper, as Predelli does, you find that some expressively valid arguments are not truth-preserving (Predelli 2010: 171-172 and 2013: 119-121). To illustrate Predelli's findings, let ' $\vDash$ ' stand for ordinary semantic entailment (based on truth preservation) and let ' $\models_{e x}$ ' stand for expressive consequence relation:

(16) Max is a Frenchman $\vDash$ Max is a Frog.

(17) Max is a Frenchman $\sharp_{e x}$ Max is a Frog.

6 He does this for "argument's sake" because his conclusion is that validity-plus does not work. 
(18) Max is a Frog $\#$ Max is a Frenchman \& I regard the French as vulgar.

(19) Max is a Frog $\vDash_{e x}$ Max is a Frenchman \& I regard the French as vulgar.

(16) is truth-preserving since 'Frenchman' and 'Frog' are truth-conditionally indistinguishable. As far as ordinary truth preservation is concerned, (16) and the converse (Max is a Frog $\vDash$ Max is a Frenchman) are equally valid. ${ }^{7}$ However, the expressive element blocks the expressive validity of the inference in (17). The pair (16) and (17) shows that there are arguments that are truth-preserving but not expressively valid. This is compatible with Kaplan's notion of validity-plus. The pair (18) and (19) shows that there are arguments that are expressively valid but not truth-preserving. This goes against validity-plus. In this case, it is clear that we need to distinguish two notions of validity: ordinary validity based on truth preservation and expressive validity. These notions do not have the kind of relation that Kaplan had in mind (Predelli 2010: 170-172).

\section{A challenge for semantic innocence}

As a result, Predelli rejects the idea of a single validity-plus. Instead, he makes a distinction between the two notions of validity. This distinction plays a crucial role in the argument against semantic innocence. A proponent of validity-plus says that slurs should be given an expressive treatment, and thus, validity-plus should be applied arguments involving slurs. Hom and May may grant that validity-plus could be applied to arguments containing expressives like 'ouch', 'damn', 'alas' and 'hurray', but at the same time, they deny that it does not apply slurs since slurs do not contain expressive elements. Given Hom and May's lexical analysis, slurs should be treated truth-conditionally, and as it happens, the truth conditions of unnegated slurring statements are systematically false. ${ }^{8}$ It seems to me that the proponent of validity-plus and Hom and May are talking past each

7 The previous pair

(5) Max is a Boche \& Boche $=$ German. Thus, Max is a German.

(6) Max is a German \& Boche $=$ German. Thus, Max is a Boche.

illustrate this.

8 In “Ouch and Oops”, Kaplan mainly talks about words like 'ouch', 'oops' and 'damn' but he does mention 'Honkey' which is a slur for white people. Predelli explicitly expands Kaplan's insights to slurs. Hom and May could admit that Kaplan is right about 'ouch' and 'oops' but, at the same time, deny the expansion to slurs as misguided since they believe that the derogation associated with slurs should be treated truth-conditionally. 
other. For this reason, Predelli's observation is critical. Kaplanian inferences has to satisfy two notions of validity: ordinary validity based on truth preservation and expressive validity. When we consider the non-xenophobic understanding of (3b), ordinary truth preservation and expressive validity are equally important. The understanding of (3b) requires both. This is bad news for semantic innocence. Hom and May might have a legitimate reason to deny expressive validity. After all, they deny that slurs contain expressive elements, but given Predelli's observation, they still owe us an explanation concerning ordinary truth preservation.

\section{Conclusion}

Predelli's distinction between the notions of validity can accommodate the intuition concerning the use of slurs quite well. Take the contrast between (16) and (17) and you will notice that, for (16), the counterexamples are the contexts in which the speaker does not have the derogatory attitude. The most common context is one in which the speaker is incompetent. She does not know the slur expresses speaker's hostile attitude. It has to be emphasised that incompetent uses are not the same as appropriate uses. A non-xenophobic but incompetent user does not want to express xenophobic or racist beliefs but the speaker does not know that the derogatory element is part of the meaning of the word. The incompetence then stems from the speaker's ignorance that a word she used expresses racist beliefs. This is one say state the autonomy of derogation. Derogation is autonomous from anyone's intentions or beliefs. Rather, it is part of the meaning of slurs. The use of slurs does not logically entail the speaker's racist attitude. However, expressively it does. The upshot is that expressive validity requires that the derogatory element in slurs is an autonomous part of the meaning. Expressive validity involves the derogatory attitude towards the French in the meaning of 'Frog'. Observe the modified discussion between Jill and Mary:

(20) Jill: Yes, there were a few Boches and a Frog.

(21) Mary: Jesus, don't be a racist!

The response is based on Mary's competency. As far as truth preservation is concerned, Mary understands that Jill is talking about the Germans and the French but as a competent speaker, Mary also understands that 'Boche' displays a xenophobic attitude towards the Germans and 'Frog' displays a xenophobic attitude towards the French. This is the expressive dimension of slurs that is not captured 
by truth preservation. Rather, it is captured by expressive validity. The important point is that both ordinary truth preservation and expressive validity are constrained by the meaning of slurs. If Jill also is a competent speaker, then (20) and (21) illustrate a genuine conflict between xenophobic and non-xenophobic attitudes. If it turned out that Jill was in fact incompetent, Mary would still be entitled to her anti-racist reaction. The entitlement stems from the meaning of 'Boche' and 'Frog'. In the case of incompetency, it would seem that Jill owes an apology, not for being a xenophobe, but for using the terms incorrectly (see also Bolinger 2018: 441-442). Crucially, with the notion of expressive validity, we can see how the meaning of slurs constrains inferences containing slurs as seen above.

As a result of his treatment of slurs, Predelli explicitly rejects Hom and May's view that the mere use of slurs is a sign of incompetency:

[N]otwithstanding the confused and unpleasant attitude apparently conveyed by uses of slurs [...], these uses may hardly qualify as linguistically defective. For one thing, racist and xenophobic attitudes are empirically incorrect [...] (Predelli 2010: 180.)

I agree with Predelli. Although it is a tempting idea to attribute linguistic confusion to racism and xenophobia, the current conclusion suggests that that would be a mistake. It seems to me that the racists and the xenophobes are very capable in expressing their hateful and empirically incorrect thoughts. In this sense, the xenophobes and the racists use slurs correctly, and we understand their expressions of hatred. Hom and May's claim that racist and xenophobic attitudes are not only empirically false but the expressions of those attitudes with slurs are also linguistically confused is too strong. Earlier, this point was labelled as asymmetry between the xenophobic and the non-xenophobic understanding. To maintain the asymmetry, you have to attribute a linguistic confusion to xenophobia and racism. Ironically, this leads to a situation where the explanation for non-xenophobic understanding is lost as the current result suggests that semantic innocence struggles to explain the non-xenophobic version of Kaplanian inferences. The idea that slurs do make sense should be applied both to the non-xenophobic and to the xenophobic understanding. As Simon Blackburn points out, "bad people moralise badly but they need not be confused” (Blackburn 1984: 184). In this sense, you should not underestimate your opponent in the struggle against racism and xenophobia.

\section{References}

Blackburn, Simon. 1984. Spreading the word. Oxford: Oxford University Press. Bolinger, Renée. 2017. The pragmatics of slurs. NOÛS 51(3). 439-462. 
Cepollaro, Bianca, and Isidora Stojanovic. 2016. Hybrid evaluatives: In defense of a presuppositional account. Grazer Philosophische Studien 93. 458-488.

Frege, Gottlob. 1964. The basic laws of arithmetic: Exposition of the system. Berkeley, CA: University of California Press.

Hom, Christopher. 2012. A puzzle about pejoratives. Philosophical Studies 159. 383-405.

Hom, Christopher. 2008. The semantics of racial epithets. The Journal of Philosophy 105. 416-440.

Hom, Christopher, and Robert May. 2013. Moral and semantic innocence. Analytic Philosophy 54. 293-313.

Jeshion, Robin. 2013. Slurs and stereotypes. Analytic Philosophy 54. 314-329.

Kaplan, David. 2004. The meaning of 'Ouch' and 'Oops'. Unpublished manuscript. http://eecoppock.info/PragmaticsSoSe2012/kaplan.pdf (checked August 23, 2018).

Kaplan, David. 1989. Demonstratives. In J. Almog, J. Perry \& H. Wettstein (eds.), Themes from Kaplan, 483-563. Oxford: Oxford University Press.

McCready, Eric. 2010. Varieties of conventional implicature. Semantics \& Pragmatics 3. 1-57.

Potts, Christopher. 2007. The expressive dimension. Theoretical Linguistics 33(2). 165-198.

Predelli, Stefano. 2013. Meaning without truth. Oxford: Oxford University Press.

Predelli, Stefano. 2010. From the expressive to the derogatory. In S. Sawyer (ed.), New waves in philosophy of language, 164-185. Basinstoke: Palgrave Macmillan.

Williamson, Timothy. 2009. Reference, inference, and the semantics of pejoratives. In J. Almog \& P. Leonardi (eds.), The philosophy of David Kaplan, 137-158. Oxford: Oxford University Press. 